\title{
Fifth supplement to the catalogue of observed periods of Ap stars ${ }^{\star}$
}

\author{
P. Renson ${ }^{1}$ and F. A. Catalano ${ }^{2}$ \\ 1 Institut d'Astrophysique, Université de Liège, Avenue de Cointe 5, 4000 Liège, Belgium \\ e-mail: renson@astro.ulg.ac.be \\ 2 Dipartimento di Fisica e Astronomia dell'Università di Catania, Città Universitaria, via S. Sofia, \\ and C.N.R.-G.N.A., Unità di Ricerca di Catania, 95125 Catania, Italia
}

Received 2 May 2001 / Accepted 24 July 2001

\begin{abstract}
New data on the periods of Ap stars with references are presented. 161 further stars are introduced for which a periodic variability has recently been discovered or it was known before but it was not reported in previous issues of this catalogue. For many stars also present in previous issues of the catalogue new determinations of the periods are given. Recently attributed variable star names are also quoted.
\end{abstract}

Key words. catalogs - stars: chemically peculiar - stars: early-type - stars: variables: general

\section{Introduction}

Several new or improved data have appeared in the literature in the last three years to make the updating of the catalogue of observed periods of Ap stars useful.

Some of the results reported here simply confirm or improve a previously known value of the period on the basis of new observations. This is very important for planning observations. However most periods' values are from the HIPPARCOS survey (henceforth referred to simply as Hipparcos).

We also report here the variable star names which have been attributed since the publication of the previous update of our catalogue.

\section{The Catalogue}

The presentation of the data is the same as that adopted in the main catalogue and the supplements (Catalano \& Renson 1984, 1988, 1997; Catalano et al. 1991, 1993).

The stars which appear here for the first time are marked with a + before their HD number: we present here 161 such stars, of which 155 are present in Table 1, 1 in Table 2 and 5 in Table 3.

Send offprint requests to: F. A. Catalano,

e-mail: fcatalano@ct.astro.it

* Tables 1 to 3 are only available in electronic form at the CDS via anonymous ftp to

cdsarc.u-strasbg.fr (130.79.128.5) or via

http://cdsweb.u-strasbg.fr/cgi-bin/qcat?J/A\&A/378/113
As in the previous issues of the catalogue we have marked by asterisks the presence of notes; a double asterisk indicates a new or modified note. Notes are here reported for the newly introduced stars marked with an asterisk and for those marked with a double one. For the stars which were quoted in the previous issues (one asterisk), we do not report here the list of the notes: they can be easily found in Catalano \& Renson (1984, 1988, 1997) and in Catalano et al. (1991, 1993).

In the tables some stars are present without references: this means that a variable star name or a new Rns number has been recently attributed or a correction has been introduced, or a new or a modified note has been introduced.

Finally we want to point out that the periods listed in the present supplement, as in the previous issues, are physically linked only with axial rotation (via the oblique rotator model) or eclipses, hence we have not included the very short periods, i.e. those attributed to pulsations or oscillations.

Some stars among the newly introduced ones do not have any HD number (one of them has an HDE number); they are found at the place corresponding to their Rns number, i.e. to their 1900.0 coordinates.

We wish to ask our colleagues working on all aspect of Ap stars to send to F. A. Catalano or P. Renson theirs results. This would make the task of continuous updating the catalogue easier. 
Acknowledgements. Our thanks are due to the colleagues who have kindly supplied us their results.

\section{References}

Adelman, S. J. 1997a, PASP, 109, 9

Adelman, S. J. 1997b, A\&AS, 122, 249

Adelman, S. J. 1997c, A\&AS, 125, 65

Adelman, S. J. 1998, A\&AS, 128, 245

Adelman, S. J. 1999, A\&AS, 134, 53

Adelman, S. J. 2000a, A\&A, 357, 548

Adelman, S. J. 2000b, A\&AS, 146, 13

Adelman, S. J. 2001, A\&A, 368, 225

Adelman, S. J., \& Brunhouse, E. F. 1998, PASP, 110, 1304

Adelman, S. J., \& Rice, R. H. 1999, A\&AS, 136, 111

Adelman, S. J., Pi, C.-L. M., \& Rayle, K. E. 1998, A\&AS, 133, 197

Adelman, S. J., Rayle, K. E., \& Pi, C.-L. M. 1999, A\&AS, 136, 379

Babel, J., \& North, P. 1997, A\&A, 325, 195

Bagnulo, S., \& Landolfi, M. 1999, A\&A, 346, 158

Bagnulo, S., Landolfi, M., \& Landi Degl'Innocenti, M. 1999, A\&A, 343, 865

Bolton, C. T., Harmanec, P., Lyons, R. W., Odell, A. P., \& Pyper, D. M. 1998, A\&A, 337, 183

Brodskaya, E. S. 1978, Perem. Zvezdy, 20, 517

Burke, E. W. Jr., \& Barr, T. H. 1981, PASP, 93, 344

Bychkov, V. D. 1998, Bull. Crimean Astrophys. Obs., 94, 202

Bychkov, V. D., \& Shtol, V. G. 1997, New period for the magnetic star $\gamma$ Equ, in Stellar magnetic fields, Proc. Int. Conf. held at Special Astrophys. Obs., ed. Yu. V. Glagolevskij, \& I. I. Romanyuk (Russian Academy of Sciences), 200

Bychkov, V. D., Kostynchuk, L. Yu., \& Shtol, V. G., 1997a, Magnetic field variability of HD 96707, in Stellar magnetic fields, Proc. Int. Conf. held at Special Astrophys. Obs., ed. Yu. V. Glagolevskij, \& I. I. Romanyuk (Russian Academy of Sciences), 110

Bychkov, V. D., Shtol, V. G., \& Hubrig, S. 1997b, Possible presence of a weak magnetic field in $\mathrm{HgMn}$ star $33 \mathrm{Gem}$, in Stellar magnetic fields, Proc. Int. Conf. held at Special Astrophys. Obs., ed. Yu. V. Glagolevskij, \& I. I. Romanyuk (Russian Academy of Sciences), 197

Bychkov, V. D., Gerth, E., Kroll, R., \& Shtol, V. G. 1997c, Long-period magnetic variability of HD 9996, in Stellar magnetic fields, Proc. Int. Conf. held at Special Astrophys. Obs., ed. Yu. V. Glagolevskij, \& I. I. Romanyuk (Russian Academy of Sciences), 204

Bychkov, V. D., Shtol, V. G., Hubrig, S., Gerth, E., \& Kroll, R. 1996, Special Astrophys. Obs. Rep., 46

Catalano, F. A., \& Renson, P. 1984, A\&AS, 55, 371

Catalano, F. A., \& Renson, P. 1988, A\&AS, 72, 1

Catalano, F. A., \& Renson, P. 1997, A\&AS, 121, 57

Catalano, F. A., Leone, F., \& Kroll, R. 1998a, A\&AS, 129, 463

Catalano, F. A., Leone, F., \& Kroll, R. 1998b, A\&AS, 131, 63

Catalano, F. A., Renson, P., \& Leone, F. 1991, A\&AS, 87, 59

Catalano, F. A., Renson, P., \& Leone, F. 1993, A\&AS, 98, 269

Catanzaro, G., Leone, F., \& Catalano, F. A. 1999, A\&AS, 134, 211

Clausen, J. V., Giménez, A., \& van Houten, C. J. 1995, A\&AS, 109,425

de Landtsheer, A. C. 1983, A\&AS, 53, 161

Duerbeck, H. W., \& Schettler, A. 1979, Acta Astr., 29, 225

Gerth, E., Glagolevskij, Yu. V., Hildebrandt, G., Lehmann, H., \& Scholz, G. 1999, A\&A, 351, 133
Gieseking, F. 1979, A\&AS, 36, 37

Gonzalez, J.-E., \& Artru, M.-C. 1994, A\&A, 289, 209

Guthnik, P. 1942, Abh. Preuss. Akad. Wissenschaften No.7

Hill, G. M., Aikman, G. C. L., Cowley, A. P., Bolton, C. T., \& Thomas, J. C. 1976, ApJ, 208, 152

Hill, G. M., Bohlender, D. A., Landstreet, J. D., et al. 1998, MNRAS, 297, 236

Hipparcos Catalogue 1997, ESA SP-1200

Hube, D. P., \& Aikman, G. C. L. 1991, PASP, 103, 49

Jerzykiewicz, M. 1993, A\&AS, 97, 421

Kurtz, D. W., Marang, F., van Wyk, F., \& Roberts, G. 1996, MNRAS, 280, 1

Kurtz, D. W., van Wyk, F., Roberts, G., Marang, F., et al. 1997, MNRAS, 287, 69

Kuschnig, R., Ryabchikova, T. A., Piskunov, N. E., Weiss, W. W., \& Gelbmann, M. J. 1999, A\&A, 348, 924

Lehmann, H., Scholz, G., Hildebrandt, G., \& Panov, K. 1999, A\&A, 351, 267

Leone, F., \& Catanzaro, G. 2001, A\&A, 365, 118

Leone, F., Catanzaro, G., \& Catalano, S. 2000a, A\&A, 355, 315

Leone, F., Catanzaro, G., \& Malaroda, S. 2000b, A\&A, 359, 635

Leroy, J. L., Bagnulo, S., Landolfi, M., \& Landi Degl'Innocenti, E. 1994, A\&A, 284, 174

Malanushenko, V. P. 1996, Astrophys., 39, 208

Manfroid, J., Burnet, M., \& Renson, P. 1998, A\&AS, 127, 201

Manfroid, J., \& Mathys, G. 1997, A\&A, 320, 497

Manfroid, J., \& Mathys, G. 2000, A\&A, 364, 689

Manfroid, J., \& Renson, P. 1983, A\&AS, 51, 267

Mathys, G., \& Hubrig, S. 1997, A\&AS, 124, 475

Mathys, G., Hubrig, S., Landstreet, J. D., Lanz, T., \& Manfroid, J. 1997, A\&AS, 123, 353

Mathys, G., \& Lanz, T. 1997, A\&A, 323, 881

Mathys, G., Manfroid, J., \& Renson, P. 1986, A\&AS, 63, 403

Nordström, B., \& Johansen, K. T. 1994, A\&A, 282, 787

Pyper, D. M., Ryabchikova, T., Malanushenko, V., et al. 1998, A\&A, 339, 822

Renson, P. 1997, Inf. Bull. Var. Stars, 1280

Renson, P., Manfroid, J., \& Heck, A. 1976, A\&AS, 23, 413

Rice, J. B. 1988, A\&A, 199, 299

Romanyuk, I. I., Elkin, V. G., Wade, G. A., \& Landstreet, J. D. 1997, The very strong and complex magnetic field of the helium-strong star HD 37776, in Stellar magnetic fields, Proc. Int. Conf. held at Special Astrophys. Obs., ed. Yu. V. Glagolevskij, \& I. I. Romanyuk (Russian Academy of Sciences), 101

Ryabchikova, T. A., Malanushenko, V. P., \& Adelman, S. J. 1999, A\&A, 351, 963

Scholz, G., Gerth, E., \& Panov, K. P. 1985, Astr. Nachr., 306, 329

Scholz, G., Hildebrandt, G., Lehmann, H., \& Glagolevskij, Yu. V. 1997, A\&A, 325, 529

Shtol, V. G., Elkin, V. G., \& Romanyuk, I. I. 1997, Magnetic field of the star $\varepsilon$ UMa $=$ HD 112185, in Stellar magnetic fields, Proc. Int. Conf. held at Special Astrophys. Obs., ed. Yu. V. Glagolevskij, \& I. I. Romanyuk (Russian Academy of Sciences), 207

Sokolov, N. A. 1999, Inf. Bull. Var. Stars, 4781

Sokolov, N. A. 2000, A\&A, 353, 707

Wade, G. A., Elkin, V. G., Landstreet, J. D., Leroy, J.-L., Mathys, G., \& Romanyuk, I. I. 1996a, A\&A, 313, 209

Wade, G. A., North, P., Mathys, G., \& Hubrig, S. 1996b, A\&A, 314,491 
Wade, G. A., Bohlender, D. A., Brown, D. N., et al. 1997a, A\&A, 320, 172

Wade, G. A., Elkin, V. G., Landstreet, J. D., \& Romanyuk, I. I. 1997b, MNRAS, 292, 748

Wade, G. A., Hill, G. M., Adelman, S. J., Manset, N., \& Bastien, P. 1998, A\&A, 335, 973

Wade, G. A., Mathys, G., \& North, P. 1999, A\&A, 347, 164

Wade, G. A., Donati, J.-F., Landstreet, J. D., \& Shorlin, S. L. S. 2000a, MNRAS, 313, 851

Wade, G. A., Kudryavtsev, D., Romanyuk, I. I., Landstreet, J. D., \& Mathys, G. 2000b, A\&A, 355, 1080
Wade, G. A., Debernardi, Y., Mathys, G., et al. 2000c, A\&A, 361, 991

Weiss, W. W., Kuschnig, R., Mkrtichian, D. E., et al. 1998, A\&A, 338, 919

Yushenko, A. V., Gopka, V. F., Khokhlova, V. L., Musaev, F. A., \& Bikmaev, I. F. 1999, Astron. Lett., 25, 453

Zakirov, M. M., \& Arzumanyants, G. C. 1995, Inf. Bull. Var. Stars, 4280

Z̆ižn̆ovský, J., Schwartz, P., \& Zverko, J. 2000, Inf. Bull. Var. Stars, 4835 